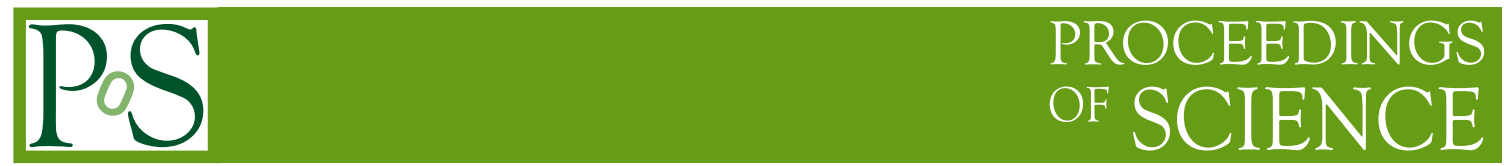

\title{
Constraints on minimal Z'models from SO(10) GUTs
}

\author{
Paweł PACHOŁEK* \\ University of Warsaw \\ E-mail: pawel.pacholekefuw.edu.pl
}

\begin{abstract}
We show how to embed a theory with the $Z^{\prime}$ boson (gauge boson of extra $U(1)$ gauge group) into a GUT theory based on $S O(10)$. Two embeddings with two different sequences of $S O(10)$ breaking are shown. Unification of gauge coupling constants provides constraints on the mass of the $Z^{\prime}$ boson and on the low energy values of two additional gauge coupling constants related to $Z^{\prime}$ interactions with fermions. Analytic formulas for these constraints have been derived at 1-loop level. Unification constraints are compared to the latest results from the LHC and electroweak precision tests (EWPT).
\end{abstract}

Proceedings of the Corfu Summer Institute 2011 School and Workshops on Elementary Particle Physics and Gravity

September 4-18, 2011

Corfu, Greece

\footnotetext{
* Speaker.
} 


\section{Introduction}

The minimal Z' model is a model with the gauge group $G_{S M} \oplus U(1)$, where $G_{S M}$ is the Standard Model Gauge Group. It can be also written as $S U(3)_{c} \oplus S U(2)_{L} \oplus U(1)^{2}$. The word "minimal" means, that no fermions, which are additional with respect to the SM (except for RH-neutrinos), are needed for anomaly cancellation. This is possible only when the $U(1)^{2}$ algebra is spanned by the weak hypercharge $(Y)$ and $B-L$ generators. The $Z$ ' boson is by definition the gauge boson of additional $U(1)$ gauge group.

The model has three basic parameters, additional with respect to the SM - the mass of the $Z^{\prime}$ boson - $M_{Z^{\prime}}$ and two additional gauge coupling constants $-g_{B-L}^{\prime}$ and $g_{B-L}$. In the general class of $Z^{\prime}$ models, they are free parameters, that are constrained only by experiments (the LHC and electroweak precision tests from LEP). After embedding a $Z^{\prime}$-model into a GUT model, unification of gauge coupling constants provides additional constraints. They can be added to experimental ones, which allowes to obtain a lower limit for $M_{Z^{\prime}}$ for each type of embedding.

The paper is organized as follows. Section 1 contains basic information about minimal $Z^{\prime}$ models including the Standard Parametrization. In Section 2 we show two specific, supersymmetric $S O(10)$-GUT models, that contain minimal Z' models as the low-energy limits. Each of them is based on a different pattern of gauge symmetry breaking. Section 3 is dedicated to the procedure of obtaining constraints on couplings $g_{B-L}^{\prime}$ and $g_{B-L}$ from analytic, 1-loop solutions of gauge RGE. Results of this procedure, applied to models 1 and 2, are compared with experimental constraints in Section 4. Then, we conclude in Section 5.

\section{Minimal Z' models - formalism and Standard Parametrization}

The most useful form of the Lagrangean for a theory with unbroken $U(1)^{2}$ gauge symmetry contains the following, abelian part

$$
\begin{aligned}
L^{\text {abelian }}= & -\frac{1}{4} F_{a}^{\mu v} F_{a \mu v}+i \sum_{f} \bar{\Psi}_{f} \gamma_{\mu}\left(\partial^{\mu}-i\left(X^{T}\right)_{a}^{f} G_{a b} A_{b}^{\mu}\right) \Psi_{f} \\
& +\sum_{s}\left[\partial^{\mu} \varphi_{s}-i\left(\left(X^{T}\right)_{a}^{s} G_{a b} A_{b}^{\mu}\right) \varphi_{s}\right]\left[\partial_{\mu} \varphi_{s}^{*}+i\left(\left(X^{T}\right)_{c}^{s} G_{c d} A_{\mu d}\right) \varphi_{s}^{*}\right]
\end{aligned}
$$

Small latin index $s$ denotes a single complex scalar field. Analogicaly, small latin index $f$ denotes a single fermionic (Weyl) field. $\Psi_{f}$ is a chiral field (left-handed or right-handed) in Dirac notation. Small Latin indeces $a, b, c, d \in\{1,2\}$ are related to the $U(1)^{2}$ algebra. $A_{a}^{\mu}$ denotes an abelian gauge field, $F_{a}^{\mu \nu}=\partial^{\mu} A_{a}^{v}-\partial^{v} A_{a}^{\mu}, G_{a b}$ is an abelian gauge coupling constant, $X_{a}^{x}$ and $X_{c}^{x}$ are charges of a single field $x(s$ or $f)$.

There is large freedom of transformations, that preserve the form of the Lagrangean given in eq. (2.1). Firstly, one can transform fields $A_{a}^{\mu}$ orthogonaly

$$
A_{a^{\prime}}^{v}:=O_{a^{\prime} b} A_{b}^{v}
$$

where $O_{a^{\prime} b}$ is an orthogonal matrix. Secondly, one can transform abelian generators $X_{a}$ linearly

$$
X_{a^{\prime}}:=L_{a^{\prime} b} X_{b}
$$


where $L_{a^{\prime} b}$ is a linear, invertable matrix. Gauge coupling matrix $G_{a b}$ transforms under eq. (2.2) and (2.3) in the following way

$$
G_{a^{\prime} b^{\prime}}:=\left(L^{T}\right)_{a^{\prime} b}^{-1} G_{b a} O_{a b^{\prime}}^{T}
$$

One can use the above freedom to obtain the Standard Parametrization in a minimal $Z^{\prime}$ model. In this parametrization $X=\left[\begin{array}{c}Y \\ B-L\end{array}\right]$ and the freedom given by $O$-transformations (2.2) is used to demand $G_{21}=0$

$$
X_{a}^{T} G_{a b} A_{b}^{\mu}=[Y, B-L]\left[\begin{array}{ll}
g^{\prime} & g_{B-L}^{\prime} \\
0 & g_{B-L}
\end{array}\right]\left[\begin{array}{c}
B^{0 \mu} \\
Z^{\prime 0 \mu}
\end{array}\right]
$$

As one can see, the gauge boson $B^{0}$ couples only to weak hypercharge $Y$ through the $g^{\prime}$ coupling constant, exactly like in the SM. One should remember, that $Z^{\prime 0}$ is not the final $Z^{\prime}$ boson. $Z^{\prime}$ is a mass eigenstate, which is a linear combination of $W^{3}, B^{0}$ and $Z^{\prime \prime}$. The formula (2.5) is correct for energy scales higher than $M_{Z^{\prime}}$. Below this scale the $U(1)^{2}$ symmetry is broken down to $U(1)_{Y}$, so the $Z^{\prime}$ boson is integrated out and $g_{B-L}^{\prime}, g_{B-L}$ are eaten by appriopriate, dimensionfull Wilson coefficients of the effective theory.

\section{Embedding Z' models into SO(10) GUT models}

In a GUT model, in which the GUT group is broken down to the Z' gauge group $\left(S U(3)_{c} \oplus\right.$ $\left.S U(2)_{L} \oplus U(1)^{2}\right)$, there is a natural parametrization of the $U(1)^{2}$ algebra - it can be spanned by appriopriate, diagonal generators of the GUT group (let's denote them $X_{\widehat{1}}$ and $X_{\widehat{2}}$ ). This parametrization is different, than the standard one, so one needs an appriopriate $L$-transformation (2.3) to relate them to each other. Let's choose two specific patterns of $S O(10)$ breaking

$$
\text { Pattern I } \quad S O(10) \stackrel{\mu_{0}}{\longrightarrow} S U(5) \oplus U(1)_{X} \stackrel{\mu_{1}}{\longrightarrow} S U(3)_{c} \oplus S U(2)_{L} \oplus U(1)^{2}
$$

Pattern II $\quad S O(10) \stackrel{\mu_{0}}{\longrightarrow} S U(3)_{c} \oplus S U(2)_{L} \oplus S U(2)_{R} \oplus U(1)_{B-L} \stackrel{\mu_{1}}{\longrightarrow} S U(3)_{c} \oplus S U(2)_{L} \oplus U(1)^{2}$

$\mu_{0}$ and $\mu_{1}$ denote scales of symmetry breaking. In Pattern I, when $S O(10)$ is initially broken to $S U(5) \oplus U(1)_{X}$, the relation between $\left(X_{\widehat{1}}, X_{\widehat{2}}\right)$ basis and $(Y, B-L)$ basis is the following

$$
\text { Pattern I } \quad\left[\begin{array}{l}
X_{\widehat{1}} \\
X_{\widehat{2}}
\end{array}\right]=\left[\begin{array}{l}
\widehat{Y} \\
X
\end{array}\right]=\left[\begin{array}{cc}
\frac{\sqrt{15}}{5} & 0 \\
-\frac{\sqrt{10}}{5} & \frac{\sqrt{10}}{4}
\end{array}\right] \cdot\left[\begin{array}{c}
Y \\
B-L
\end{array}\right]
$$

As we can see, the weak hypercharge $Y$ is rescaled to $\widehat{Y}$ just like in the minimal GUT model, based on $S U(5)$ [1],[2]. When $S O(10)$ is initially broken to $S U(3) \oplus S U(2)_{L} \oplus S U(2)_{R} \oplus U(1)_{B-L}$, the relation between $\left(X_{\hat{1}}, X_{\hat{2}}\right)$ basis and $(Y, B-L)$ basis is the following

\section{Pattern II}

$$
\left[\begin{array}{l}
X_{\widehat{1}} \\
X_{\widehat{2}}
\end{array}\right]=\left[\begin{array}{c}
R \\
\widehat{B-L}
\end{array}\right]=\left[\begin{array}{cc}
1 & -\frac{1}{2} \\
0 & \frac{\sqrt{6}}{4}
\end{array}\right] \cdot\left[\begin{array}{c}
Y \\
B-L
\end{array}\right]
$$

$R$ is the third (diagonal) generator of $S U(2)_{R}$ and $\widehat{B-L}$ is the appriopriately rescaled $B-L$. In both patterns the intermediate group $\left(S U(5) \oplus U(1)_{X}\right.$ or $\left.S U(3) \oplus S U(2)_{L} \oplus S U(2)_{R} \oplus U(1)_{B-L}\right)$ is 
broken down to the $Z^{\prime}$ gauge group, which is then broken down to the SM group at the scale of $M_{Z^{\prime}}$. We consider two specific, supersymmetric models.

Model 1 is based on Pattern I and its higgs sector contains (chiral) 210, 54, $\overline{126}, 126$ and 10 of $S O(10)$. These representations aquire VEVs, that realize pattern I in the following way:

$$
\text { Model } 1 \quad S O(10) \stackrel{210}{\longrightarrow} S U(5) \oplus U(1)_{X} \stackrel{24_{54}}{\longrightarrow} S U(3)_{c} \oplus S U(2)_{L} \oplus U(1)^{2}
$$

and then

$$
S U(3)_{c} \oplus S U(2)_{L} \oplus U(1)^{2} \stackrel{\chi_{\overline{126}+126}}{\longrightarrow} S U(3)_{c} \oplus S U(2)_{L} \oplus U(1)_{Y} \stackrel{h_{10}}{\longrightarrow} S U(3)_{c} \oplus U(1)_{E M}
$$

$24_{54}$ is 24 of $S U(5)$, which is embedded in 54 of $S O(10) . \chi_{\overline{126}+126}$ is the $U(1)^{2}$-breaking higgs. It's a linear combination of two SM-singlets - $\chi^{-}$and $\chi^{+}$, that are parts of $\overline{126}$ and 126 of $S O(10)$ respectively. $h_{10}$ is the standard higgs, embedded in 10 of $S O(10)$.

Model 2 is based on Pattern II and its higgs sector contains two 45s, $\overline{126}, 126$ and 10 of $S O(10)$. These representations aquire VEVs, that realize pattern II in the following way:

$$
\text { Model } 2 \quad S O(10) \stackrel{45}{\longrightarrow} S U(3)_{c} \oplus S U(2)_{L} \oplus S U(2)_{R} \oplus U(1)_{B-L} \stackrel{[1,1,3]_{45}}{\longrightarrow} S U(3)_{c} \oplus S U(2)_{L} \oplus U(1)^{2}
$$

The higgs denoted by $[1,1,3]_{45}$ is a singlet under $S U(3)_{c} \oplus S U(2)_{L}$ and triplet under $S U(2)_{R}$, that is embedded in 45 of $S O(10)$. Further symmetry breakings are the same as in Model 1. Moreover, in both models, all SM fermions (and their superpartners) are embedded in three chiral 16's of $S O(10)[3]$.

We assume, that MSSM-fields, RH-neutrinos, $\chi^{-}, \chi^{+}$and superpartners of all these fields are relatively light with masses below the $\mu_{1}$ scale. They will be called "light" fields. All other fields are heavier and have masses between $\mu_{1}$ and $\mu_{0}$ scales. These assumptions are adopted for both considered models.

\section{Low energy analytic constraints from 1-loop unification of gauge coupling constants}

At one-loop there are analytic RGE-solutions for all non-abelian and also abelian gauge coupling constants [4]. Solutions expressed in the basis of $X_{\widehat{a}}$ generators are therefore explicitly equivalent to solutions writen in the low-energy basis. In this section analytic solutions of 1-loop gauge RGE are used to derive approximate low-energy constraints on additional abelian gauge coupling constants and treshold mass parameters.

Analytic 1-loop solution of RGE for a non-abelian gauge coupling constant $\alpha_{A}=\frac{1}{4 \pi} g_{A}^{2}$ is well known

$$
\alpha_{A}^{-1}\left(\mu_{x}\right)=\alpha_{A}^{-1}\left(\mu_{y}\right)-\frac{b_{A}}{2 \pi} \ln \left(\frac{\mu_{x}}{\mu_{y}}\right)
$$

Finding analogical solution for abelian gauge coupling constants requires introducing an appriopriate analog of $\alpha_{A}$, which is the following matrix

$$
\vartheta_{a b}=\frac{1}{4 \pi} G_{a c} G_{c b}^{T}
$$


$\vartheta_{a b}$ is $O$-invariant, but not $L$-invariant

$$
\vartheta_{a^{\prime} b^{\prime}}=L_{a^{\prime} b} \vartheta_{b a} L_{a b^{\prime}}^{T}
$$

Obtaining analytic 1-loop solution of RGE for abelian gauge coupling constants requires inverting the $\vartheta_{a b}$ matrix

$$
\vartheta_{a b}^{-1}\left(\mu_{x}\right)=\vartheta_{a b}^{-1}\left(\mu_{y}\right)-\frac{b_{a b}}{2 \pi} \ln \left(\frac{\mu_{x}}{\mu_{y}}\right)
$$

$\mu_{x}$ and $\mu_{y}$ are some energy scales $\left(\mu_{x} \geq \mu_{y}\right)$. One can apply $L_{a^{\prime} b}$ transformation to matrices in formula (4.4) (and to the whole formula itself) to write them in different basis of $X_{a^{\prime}}$ generators. In particular, in the Standard Parametrization $\vartheta_{a b}^{-1}$ has the following form

$$
\vartheta_{a b}^{-1}=4 \pi\left[\begin{array}{cc}
\frac{1}{g^{\prime 2}} & -\frac{g_{B-L}^{\prime}}{g_{B-L}} \frac{1}{g^{\prime 2}} \\
-\frac{g_{B-L}^{\prime}}{g_{B-L}} \frac{1}{g^{\prime 2}} & \frac{1}{g_{B-L}^{2}}+\left(\frac{g_{B-L}^{\prime}}{g_{B-L}}\right)^{2} \frac{1}{g^{\prime 2}}
\end{array}\right]
$$

Moreover, in the $\left(X_{\widehat{1}}, X_{\widehat{2}}\right)$ basis, at the $\mu_{1}$ scale, $G_{a b}$-matrix has to be diagonal, so matrices $\vartheta_{a b}$ and $\vartheta_{a b}^{-1}$ also have to be diagonal.

Analytic solutions of type (4.1) and (4.4) should be modified by including treshold corrections. System of all these equations should be also supplemented with unification conditions and inequalities, that originate from various constraints (perturbativity, $\mu_{0} \leq M_{P l}$, non-observation of proton decay[5], etc.). Masses of "light" fields are additionaly constrained. Effective treshold mass parameters, which are weighted (weights proportional to dynkin indeces), geometrical averages of these masses, are assumed to be larger than $1 \mathrm{TeV}$ and smaller than $10 \mathrm{TeV}$. This range has been chosen to cover typical values of the SUSY-breaking scale $T_{S U S Y}$, which can be identified with one of effective treshold mass parameters (we have chosen the one, that affects the running of $g^{\prime}$ ). It's important, whether $T_{S U S Y}$ is larger or smaller than $M_{Z^{\prime}}$, so these two cases are considered separately.

In both cases, after collecting all constraints into one big system of equations and inequalities, one can use all equations to eliminate some unwanted parameters. The result is a system, that contains only inequalities with smaller number of parameters. For a given value of $M_{Z^{\prime}}$ and in appriopriate coordinates, it defines the allowed multidimensional polyhedron in the parameterspace. One can project this polyhedron to the 2D-plane spanned by $g_{B-L}^{\prime}$ and $g_{B-L}$ only (values at the $M_{Z^{\prime}}$-scale). Results of this procedure for Models 1 and 2 are shown in the next section.

\section{Comparison between theoretical and experimental constraints}

Important experimental constraints on the minimal Z' model are currently provided not only by the LHC, but also by electroweak precision tests (EWPT) made in LEP. They have been shown as constraints on $g_{B-L}^{\prime}$ and $g_{B-L}$ for different values of $M_{Z^{\prime}}$ in [4]. We follow the convention of this paper and divide $g_{B-L}^{\prime}$ and $g_{B-L}$ by $g_{Z}$ (taken at the $M_{Z}$-scale)

$$
\widetilde{g}_{B-L}=\frac{g_{B-L}^{\prime}}{g_{Z}} \quad \widetilde{g}_{B-L}=\frac{g_{B-L}}{g_{Z}}
$$

The crucial LHC data are taken from the 95\% C.L. exclusion plot published by ATLAS collaboration [6]. It shows an upper limit for the total cross-section in the $Z^{\prime} \longrightarrow l^{+} l^{-}$channel, where $l$ is 

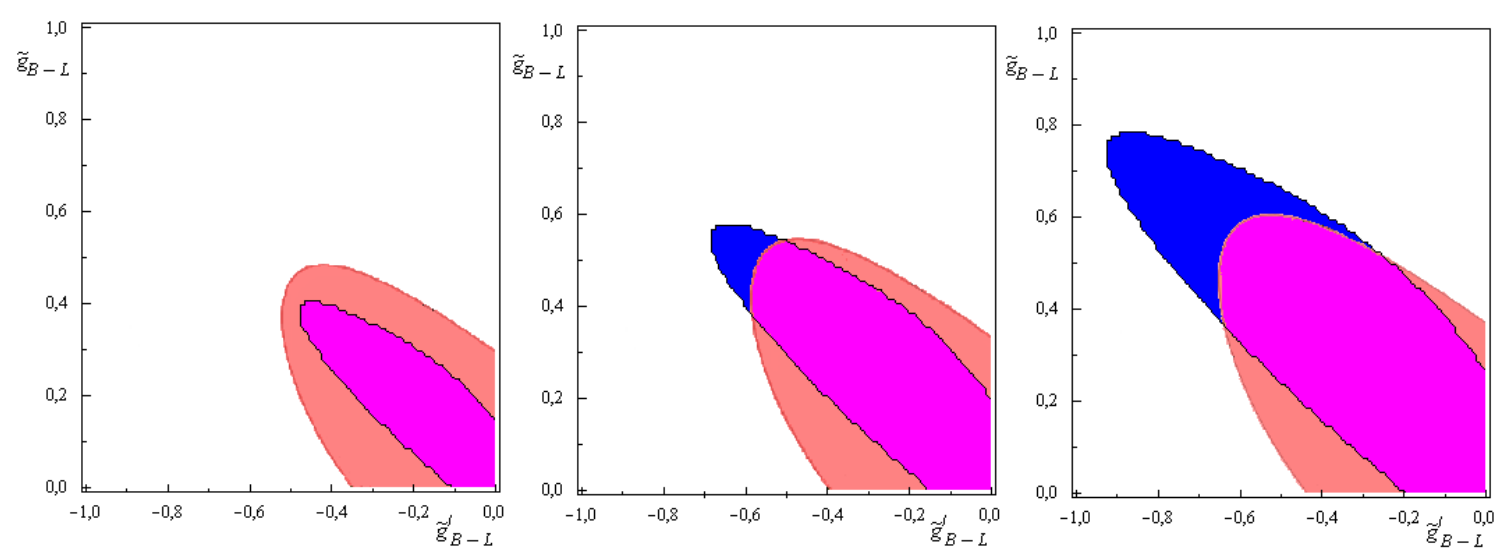

Figure 1: Experimental constraints on $\widetilde{g}_{B-L}$ and $\widetilde{g}_{B-L}$ for $M_{Z^{\prime}}$ equal to $1.6 \mathrm{TeV}$ (left plot), $1.8 \mathrm{TeV}$ (middle plot) and $2 \mathrm{TeV}$ (right plot). Blue and violet regions are allowed by ATLAS. Dark orange and violet regions are allowed by EWPT.
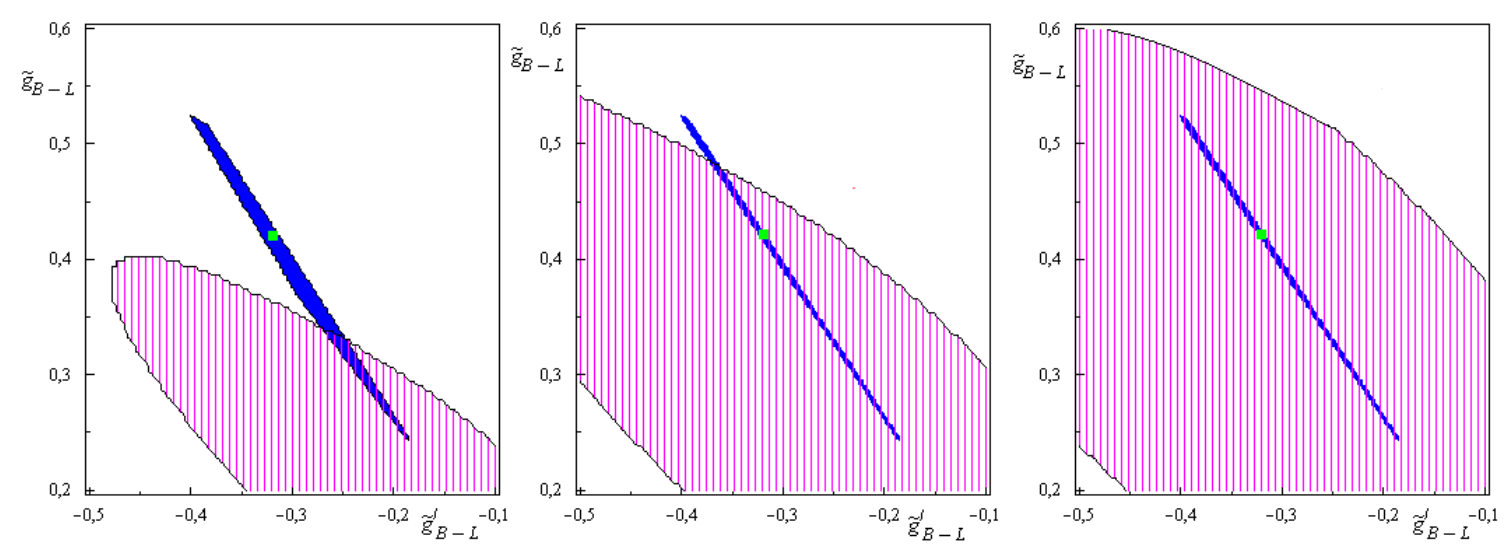

Figure 2: Comparison between unification constraints (blue region) and experimental ones (violet stripes) on $\widetilde{g}_{B-L}$ and $\widetilde{g}_{B-L}$ for Model 1 with $M_{Z^{\prime}}$ equal to $1.6 \mathrm{TeV}$ (left plot), $1.8 \mathrm{TeV}$ (middle plot) and $2 \mathrm{TeV}$ (right plot). The green square represents a small interval, which is allowed without taking into account treshold corrections.

an electron or a muon. To obtain needed constraints, we calculated this cross-section in the leading order in the narrow width approximation as a function of $\widetilde{g}_{B-L}^{\prime}$ and $\widetilde{g}_{B-L}$. Experimental constraints from ATLAS and EWPT are shown in figure 1. The procedure, described in the previous section, provides unification constraints on $\widetilde{g}_{B-L}^{\prime}$ and $\widetilde{g}_{B-L}$ in Models 1 and 2. Results are shown in figures 2 and 3 respectively.

As we can see, for $M_{Z^{\prime}}=1.6 \mathrm{TeV}$, Model 1 is strongly constrained and Model 2 is almost excluded by experiments. On the other hand, for $M_{Z^{\prime}}=2 \mathrm{TeV}$ experimental constraints are currently too weak to exclude anything in Model 1 or any significant region in Model 2. The left plot of figure 3 allows us to deduce, that the lower experimental bound on $M_{Z^{\prime}}$ in Model 2 is very close to 1.6 TeV. In Model 1, it's definitely smaller, probably in the range $1.4-1.5 \mathrm{TeV}$. 

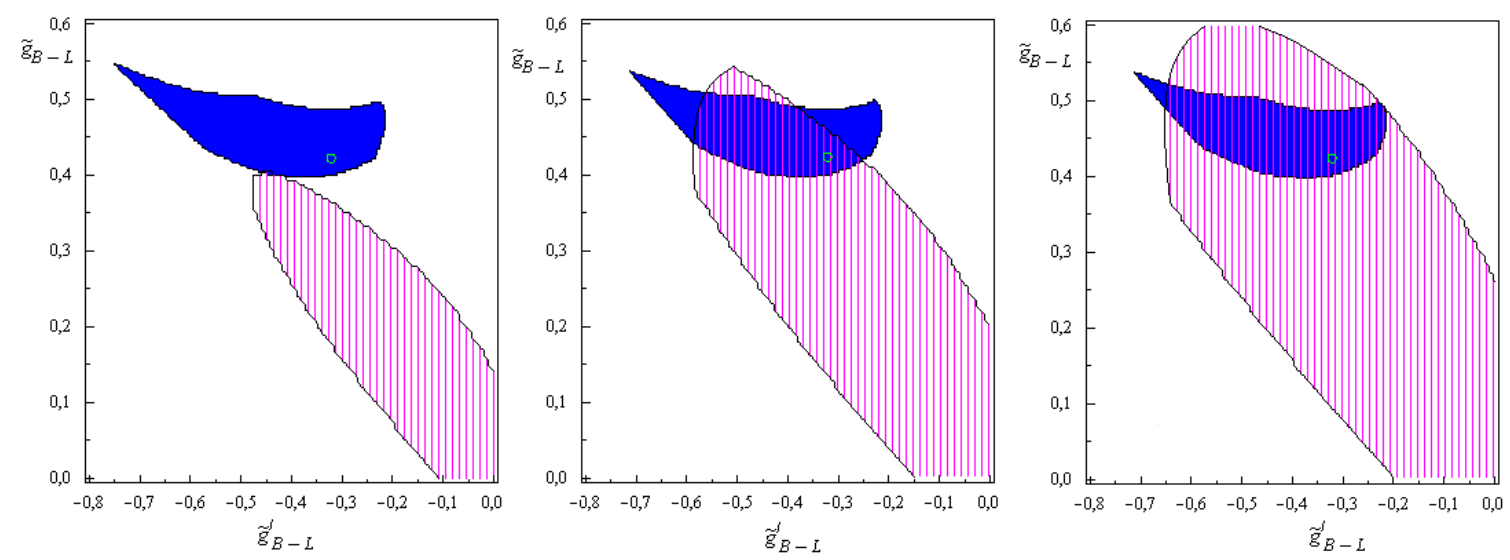

Figure 3: Comparison between unification constraints (blue region) and experimental ones (violet stripes) on $\widetilde{g}_{B-L}$ and $\widetilde{g}_{B-L}$ for Model 2 with $M_{Z^{\prime}}$ equal to $1.6 \mathrm{TeV}$ (left plot), $1.8 \mathrm{TeV}$ (middle plot) and $2 \mathrm{TeV}$ (right plot). The green circle represents a point, which is allowed without taking into account treshold corrections.

\section{Conclusions}

Constraints from Grand Unification decrease experimentally-allowed region of the parameter space in minimal $Z^{\prime}$ models. They can provide a lower bound on the $M_{Z^{\prime}}$ (like $1.6 \mathrm{TeV}$ in Model 2). Treshold corrections are important. They give additional freedom, enlarging allowed region of the parameter space. Methods presented in this paper can be used beyond minimal $Z^{\prime}$ models [7], unless there are three or more $U(1) \mathrm{s}$ at the same range of scales [8],[9].

\section{Acknowledgments}

The author thanks prof. S. Pokorski and prof. M. Olechowski for their help with this work.

\section{References}

[1] M. S. Carena, S. Pokorski, C.E.M. Wagner, On the unification of couplings in the minimal supersymmetric Standard Model, Nucl.Phys. B406:59-89, 1993 [hep-ph/9303202]

[2] J. L. Jones, Gauge Coupling Unification in MSSM + 5 Flavors, Phys.Rev. D79:075009, 2009 [arXiv:0812.2106]

[3] A. S. Joshipura, K. M. Patel Fermion Masses in SO(10) Models., Phys.Rev. D83:095002, 2011 [arXiv:1102.5148]

[4] E. Salvioni, G. Villadoro, F. Zwirner, Minimal Z-prime models: Present bounds and early LHC reach, JHEP 0911:068, 2009 [arXiv:0909.1320]

[5] G. Senjanovic Proton decay and grand unification., AIP Conf.Proc. 1200 (2010) 131-141, 2009 [arXiv:0912.5375]

[6] ATLAS-CONF-2012-007, 2012

[7] E. Salvioni, A. Strumia, G. Villadoro, F. Zwirner, Non-universal minimal Z’ models: present bounds and early LHC reach, JHEP 1003:010, 2010 [arXiv:0911.1450] 
[8] R. Slansky, Group Theory for Unified Model Building, Phys.Rept. 79:1-128, 1981

[9] F. del Aguila, G.D. Coughlan, M. Quiros, Gauge Coupling Renormalization With Several U(1) Factors, Nucl.Phys. B307:633, 1988, Erratum-ibid. B312:751, 1989

[10] K. Nakamura et al. (Particle Data Group), J. Phys. G37 075021, 2010 